\title{
Mehr Zufallsdiagnosen, neue Therapiestandards
}

— Nur bei wenigen Tumoren hat sich in den letzten Jahren so viel verändert wie beim Nierentumor. In vielen Lehrbüchern, die bei uns noch im Regal stehen, ist zu lesen, dass die führende klinische Trias "Makrohämaturie, Flankensymptome und tastbare Raumforderung in der Flanke" sei. Durch die flächendeckende Einführung des Ultraschalls und der modernen Schnittbildgebung ist diese Aussage heute unsinnig. Die allermeisten Nierentumoren werden als Zufallsbefund bei einer Bildgebung zu ganz anderen Fragestellungen entdeckt.

Auch die Behandlung hat sich grundsätzlich geändert. Bis vor wenigen Jahren war die Standardtherapie des Nierenkarzinoms die „ra-

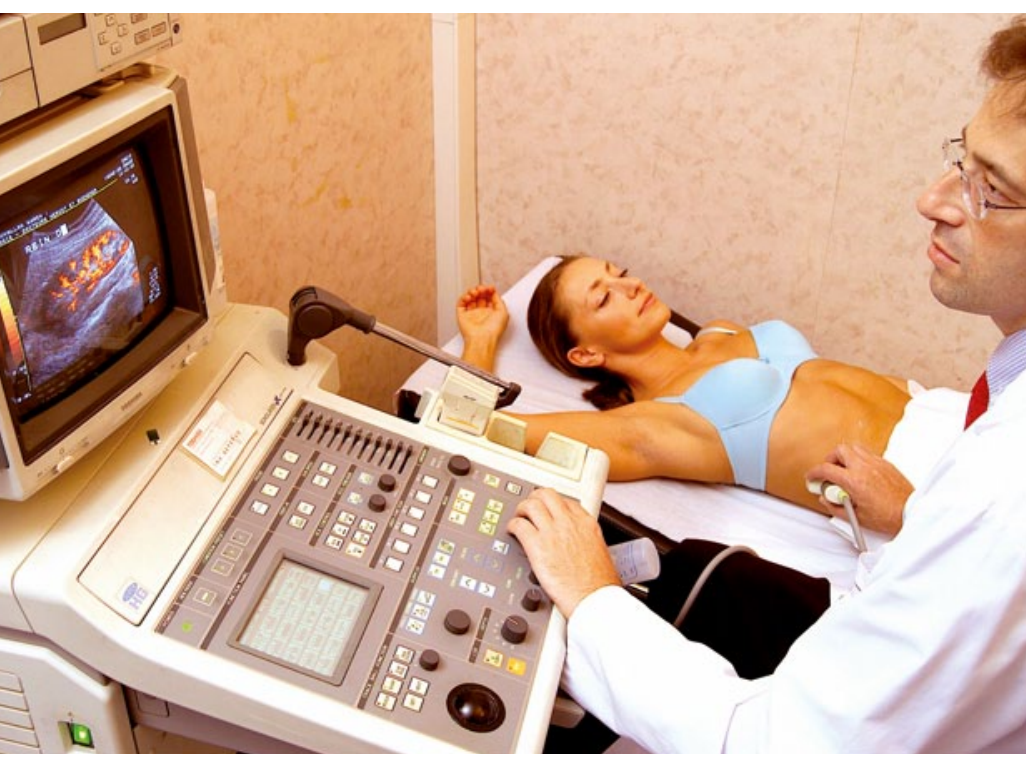

Durch die flächendeckende Einführung der Sonografie und der modernen Schnittbildgebung werden Nierentumoren heute oft zufällig entdeckt. dikale Tumornephrektomie", d.h. die komplette Entfernung der tumortragenden Niere einschließlich der ipsilateralen Nebenniere und des Nierenfetts innerhalb der Gerota-Faszie. Durch die Entdeckung der Nierentumoren als Zufallsbefund und damit kleinerer Durchmesser einerseits und die wesentliche Verbesserung der Operationsmethoden andererseits steht heute der Organerhalt im Vordergrund.

Die organerhaltende Nierentumorchirurgie („partielle Nephrektomie") ist heute der Goldstandard für ca. 80\% der Nierentumoren. Es konnte gezeigt werde, dass der Erhalt der Nierenfunktion zu einer deutlichen Verringerung der kardiovaskulären Komplikationen und zu einer Verringerung der damit verbundenen Langzeitmortalität führt. Leider zeigen epidemiologische Untersuchungen zur Häufigkeit der partiellen Nephrektomie, dass dieses zwar operativ anspruchsvolle, aber für den Patienten deutlich bessere Operationsverfahren noch nicht flächendeckend durchgeführt wird.

Bei ausgewählten Patienten (hohe Komorbidität, begrenzte Lebenserwartung) kann ein abwartendes Vorgehen („active surveillance") oder eine minimal-invasive Ablation sinnvoll sein.

Neben der Diagnostik und der Therapie des lokal begrenzten Nierentumors hat sich auch die Therapie und Prognose des fortgeschrittenen Karzinoms wesentlich verändert. 30\% der neu diagnostizierten Patienten mit Nierenkarzinom weisen - auch heute noch Metastasen auf. Die Prognose dieser Patienten war bis vor wenigen Jahren infaust mit einem Fünf-Jahre-Überleben von unter $10 \%$. Heute ist durch den gezielten Einsatz der Metastasenchirurgie und/ oder -destruktion, z. B. durch das Cyberknife und durch die „Targeted Chemotherapien", eine deutliche Verlängerung des Überlebens bei zumeist guter Lebensqualität möglich.

Zusammenfassend können wir feststellen, dass in Diagnostik und Therapie des lokalisierten und fortgeschrittenen Nierenkarzinoms innerhalb der letzten Jahre ganz entscheidende Fortschritte für unsere Patienten erzielt wurden. 